\title{
REPAIR OF LACERATED INFERIOR VENA CAVA WITH THE INTRAVASCULAR TUBE GRAFT IN REDO CARDIAC SURGERY
}

\author{
Mehmet Raşit Güney, MD, Nazan Aksoy, MD, and Özge Sezerman, MD, İstanbul, Turkey
}

Laceration of the proximal inferior vena cava (IVC) is a rare but fatal complication of redo cardiac surgery if it is situated at the posterior supradiaphragmatic, juxtadiaphragmatic, or retrohepatic region of the IVC. Redo valve and congenital cardiac operations, which are performed by the transatrial approach, may carry the risk of IVC laceration. Laceration of the IVC, mostly at its posterior wall, may occur during application of a snare or Cooley clamp or from stretching of the incompletely liberated adherent IVC during traction on the atrium for valve exposure. The laceration may extend for a variable distance down the IVC. Not infrequently, this complication may also be encountered in other cardiovascular interventions (ie, IVC filter implantation, thrombectomy), some upper abdominal operations (ie, IVC fistula repair, thoracoabdominal aneurysm surgery, hepatic surgery), and in blunt or penetrating chest traumas. Different methods have been proposed to repair this complication, such as atriocaval shunt, lateral phleborrhaphy, patch plasty, total vascular occlusion, tube graft or duplicated saphenous interposition, and diaphragmatic or pericardial flap repair. However, the inaccessible location of some lacerations and the high mortality are still a problem. ${ }^{1-5} \mathrm{We}$ are presenting a case of an IVC laceration at an inaccessible location treated by means of a novel approach.

Clinical summary. A 53-year-old woman who had a closed mitral commissurotomy operation 22 years earlier because of rheumatic valve disease was admitted to our hospital for mitral restenosis and insufficiency and 3+ tricuspid regurgitation. The operation was conducted as usual with aorta-bicaval cannulation, intermittent cold cardioplegia, and moderate hypothermia $\left(28^{\circ} \mathrm{C}\right)$. Abundant bleeding appeared around the IVC after the cannulas were drawn back into the right atrium. External interventions to stop the bleeding proved futile and total circulatory arrest had to be established. Tactile exploration from the right atrium revealed a long laceration extending down the IVC for an indeterminate distance in the

From the Siyami Ersek Thoracic and Cardiovascular Surgery Center, İstanbul, Turkey.

Received for publication May 16, 2000; accepted for publication June 19, 2000.

Address for reprints: Mehmet Raşit Güney, MD, Tütüncü Mehmet Efendi Cad. Dr Rıfatpaşa Sok. Sema Apt No 21/37 Göztepe İstanbul 81060 Turkey (E-mail: Dr.guney@ superonline.com).

J Thorac Cardiovasc Surg 2001;121:187-8

Copyright (C) 2001 by The American Association for Thoracic Surgery

0022-5223/2001 $\$ 35.00+0 \quad \mathbf{1 2 / 5 4 / 1 0 9 5 4 7}$

doi: $10.1067 / \mathrm{mtc} .2001 .109547$ extrapericardial region. It was impossible to repair the laceration by direct suturing or patch plasty. Inspired by endovascular grafting techniques, we prepared a 6 -cm long size 16 standard plain polytetrafluoroethylene tube graft* after having measured the IVC with Hegar dilators. A U-shaped indentation was created at the proximal edge of the graft to spare a high-draining hepatic vein. After being slipped over a Hegar dilatator as a guide, the graft was introduced into the IVC and fitted so that it slightly distended the IVC. The U indentation was juxtaposed to the hepatic vein ostium, and the proximal edge of the graft was circumferentially sewn to the atriocaval junction. After rewarming, cardiopulmonary bypass was terminated with trivial inotropic support. All bleeding ceased after neutralization of heparin with protamine. In the postoperative period, $900 \mathrm{~mL}$ of drainage was replaced with blood products and fresh frozen plasma. A hematoma was detected at the retroperitoneal space by ultrasonographic examination on the second postoperative day, but no intervention was necessary. The patient was discharged after an uneventful convalescence on the 10th postoperative day.

Discussion. Laceration of the proximal IVC has been encountered in 14 redo operations among more than 25,000 cardiac operations performed since 1963 by different surgeons in our hospital. Seven of 14 lacerations were simple dissection injuries and did not cause undue problems. The remaining 7 extended into inaccessible regions of the proximal IVC. From the common features of the patients, we deduced that redo operations (firm adhesions), female sex, rheumatic valve disease (pancarditis), small left atrium (more traction), and prolonged ischemic time may constitute incremental risk factors for this complication.

When an endovascular tube graft is inserted into the IVC (preferably slightly distending it), elastic recoil of the vessel may be countered by its intraluminal pressure and stiffness of the graft and thereby may effectively seal the laceration. Although the distal free edge of the graft was positioned against blood flow, the graft had not collapsed (Fig 1). The graft should be long enough to cover the laceration but not so long that it blocks the hepatic venous drainage. All of the hepatic veins were spared by the 6-cm length of the graft, as shown in this case by magnetic resonance imaging. The patient was doing well at 6 months' follow-up (Fig 2).

Although rare in cardiac surgery, laceration of the proximal IVC is not an infrequent vessel injury. This complication is especially challenging if situated in or extending to the juxtadiaphragmatic or retrohepatic portion of the IVC, since it is very hard to explore the laceration either from inside or out-

*W. L. Gore \& Associates, Inc, Flagstaff, Ariz. 


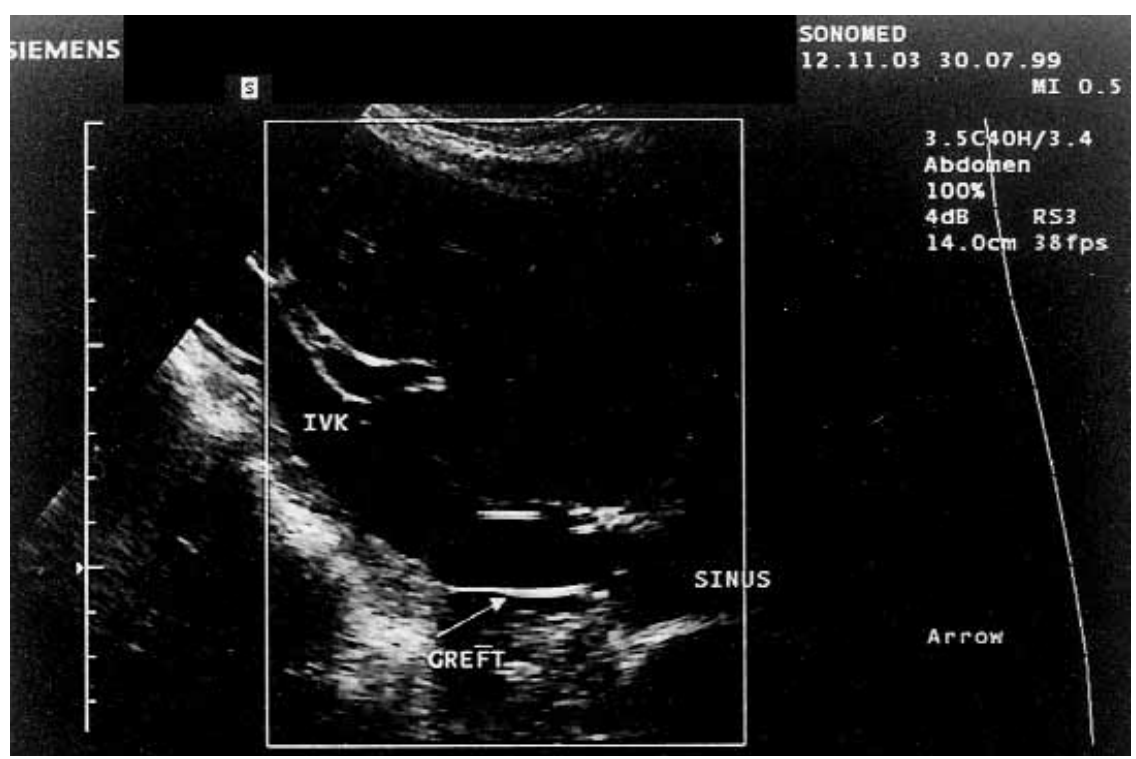

Fig 1. The ultrasound image of the IVC and the graft on postoperative day 15 . The position of tube graft can be seen to be well engaged into the IVC. The graft extends downward approximately $4.5 \mathrm{~cm}$ under the diaphragm. No residual space exists between the graft and IVC.

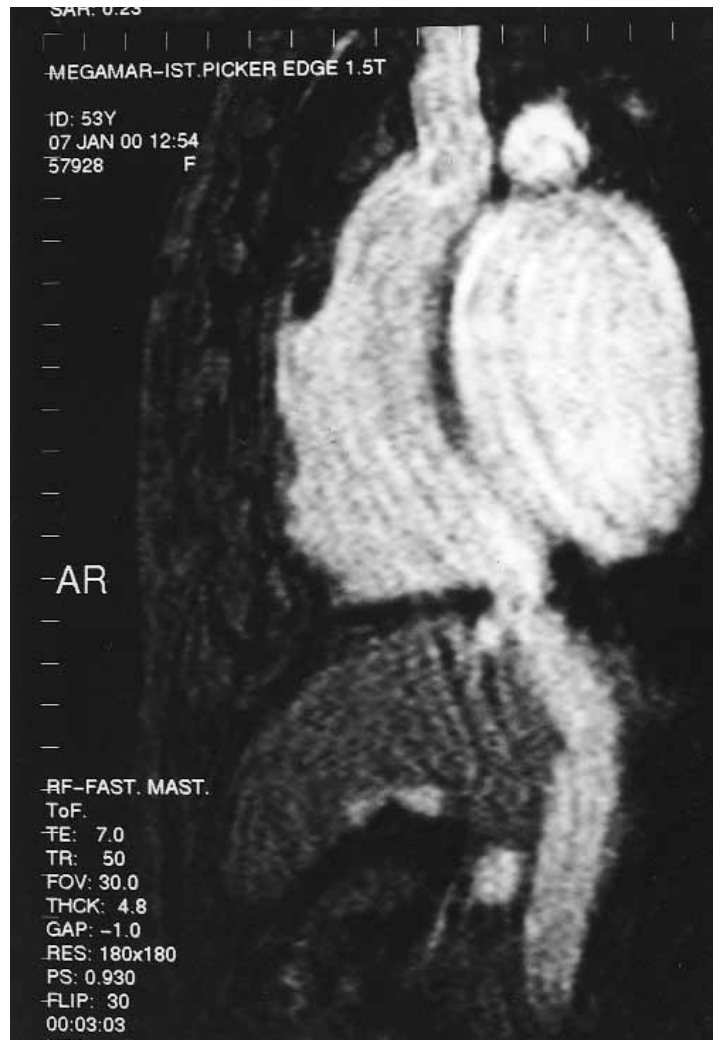

Fig 2. Magnetic resonance imaging of the grafted IVC during the sixth postoperative month. Calibration of the IVC is normal and intraluminal filling with contrast medium is homogeneous. There is no occlusion or turbulent flow pattern 6 months after the operation. side the IVC for direct suturing or patch plasty. Our novel approach may be applicable for all proximal IVC lacerations without the need to explore the laceration, and it may avoid some postoperative complications such as iatrogenic narrowing of the IVC or bleeding from a residual defect due to the inaccessible position of the laceration.

\section{REFERENCES}

1. Ombrellaro MP, Freeman MB, Stevens SL, Diamond DL, Goldman MH. Predictors of survival after inferior vena cava injuries. Am Surg 1997;63:178-83.

2. Cunningham PR, Foil MB. The ripped cava. J Natl Med Assoc 1995;87:305-7.

3. Demirtaş M, Aykut S, Dağsali S. Repair of the supradiaphragmatic inferior vena cava lacerated during redo open-heart surgery. Cardiovasc Surg 1995;3:345-7.

4. Khaneja SC, Pizzi WF, Barie PS, Ahmed N. Management of penetrating juxtahepatic inferior vena cava injuries under total vascular occlusion. J Am Coll Surg 1997;184:469-74.

5. Wiencek RG Jr, Wilson RF. Inferior vena cava injuries-The challenge continues. Am Surg 1988;54:423-8. 